\title{
Analysis and Measurement of Residual Stress in Bridge Membrane MEMS Relays
}

\author{
YONG RUAN,,${ }^{1,3}$ WEIZHONG WANG,${ }^{1}$ YONG ZHU, ${ }^{2,4}$ and ZHENG YOU ${ }^{1}$ \\ 1.-Collaborative Innovation Center for Micro/Nano Fabrication, Device and System, State Key \\ Laboratory of Precision Measurement Technology and Instruments, Department of Precision \\ Instrument, Tsinghua University, Beijing 100084, China. 2.-School of Engineering, Griffith \\ University, Nathan, Brisbane 4222, Australia. 3.-e-mail: ruanyong@mail.tsinghua.edu.cn. \\ 4.—e-mail: y.zhu@griffith.edu.au
}

\begin{abstract}
Microelectromechanical system (MEMS) relays are gradually replacing traditional relays because they are smaller and lighter and consume less power. However, performance parameters of MEMS relays, such as the pull-down voltage, response time, and resonant frequency, often deviate from those originally designed, due to residual stress generated during the fabrication process. We present herein a method to measure this residual stress, based on a metal bridge membrane MEMS relay, with the help of a nanoindenter and the finite-element method (FEM). The testing result lies in a reasonable range, indicating that this simple method is reliable and helpful for MEMS relay optimization.
\end{abstract}

Key words: MEMS relay, residual stress, nanoindenter, finite-element method (FEM)

\section{INTRODUCTION}

With the widespread use of relays in aerospace and communication applications, microelectromechanical system (MEMS) technology has shown huge development potential by combining macromachining techniques and designs for electromechanical relays with metal contacts. Compared with many other MEMS relays, the electrostatic relay has been the subject of considerable research, not having special requirements for driving materials and being easier and cheaper to fabricate. Electrostatic relays are controlled by an electrostatic force applied between the electrode plates. The performance of such relays is greatly affected by their resonant frequency, pull-down voltage, etc., necessitating measurement and analysis of residual stress, which significantly influences these two parameters. ${ }^{1,2}$ Several methods are commonly used to measure residual stress, ${ }^{3-6}$ including resonant frequency measurements, the loaded deformation method, the critical fold method, the structure displacement method, silicon bending, and Raman spectroscopy.

The generation mechanism of residual stress is discussed herein, and the load-displacement curve of a bridge membrane MEMS relay measured using a nanoindenter. Then, with the help of finiteelement analysis software (ANSYS), we simulate several mechanical performance parameters, propose an easy method to measure residual stress, and analyze the errors generated in the experiment.

\section{GENERATION OF RESIDUAL STRESS}

The metal membrane of a relay is generally fabricated by the chemical vapor deposition (CVD) method, sputtering, or electroplating. During the machining process, uneven thermal expansion, plastic deformation, doping, etc. are all responsible for lattice mismatch, leading to residual stress. There are two kinds of residual stress, viz. intrinsic and extrinsic. ${ }^{7}$ Intrinsic stress, also known as internal stress, develops during film nucleation, while extrinsic stress results from external factors, such as temperature gradients and package pressure. The generation of intrinsic stress is relatively 
complex, being studied by Windischman and Davis et al. ${ }^{8,9}$ However, a unified and systematic theory to describe it has still not been generated. This is because, under different conditions and when using different materials and technologies, the main cause of intrinsic stress may vary.

Thermal stress is a common extrinsic stress and has been studied by many researchers. It develops when a membrane is fabricated by high-temperature deposition or sputtering. This is because the thermal expansion coefficients of the membrane and substrate are different, and when cooled to room temperature, they will appear to have shrunk unevenly. The thermal expansion coefficient is an intrinsic property of a material. It may vary significantly between different materials, and even for the same material, thermal stress may also exist due to temperature gradients. It is assumed that the substrate is rigid because it is much thicker than the membrane. The strain caused by thermal expansion or contraction can then be evaluated using Eq. $1^{10}$ :

$$
\varepsilon_{\mathrm{th}}=\int_{T_{2}}^{T_{1}}\left[\alpha_{\mathrm{f}}(T)-\alpha_{\mathrm{s}}(T)\right] \mathrm{d} T,
$$

where $\alpha_{\mathrm{f}}$ and $\alpha_{\mathrm{s}}$ represent the thermal expansion coefficient of the membrane and substrate, respectively, and $T_{2}$ and $T_{1}$ are the temperature of the growing and cooled membrane. There is no stress in the direction perpendicular to the substrate, so the biaxial modulus is used in such experiments. According to Hooke's law, the thermal stress is given by

$$
\sigma_{\mathrm{th}}=\left(\frac{E}{1-v}\right) \varepsilon_{\mathrm{th}},
$$

where $E$ is the plastic modulus and $v$ is Poisson's ratio. When the temperature does not change dramatically, the thermal expansion coefficient can be treated as a constant, therefore

$$
\sigma_{\text {th }}=\left(\frac{E}{1-v}\right)\left[\alpha_{\mathrm{f}}(T)-\alpha_{\mathrm{s}}(T)\right]\left(T_{1}-T_{2}\right) .
$$

The bridge membrane MEMS relay discussed in this paper is shown in Figs. 1 and $2 .{ }^{11}$ The driving electrodes of the relay consist of an upper electrode plate (movable) and a bottom electrode plate (fixed), both of which are metal membranes to reduce the contact resistance. Damping holes are formed in the upper electrode plate to reduce the damping effect that may occur when the relay is opened or closed. After the drive voltage is applied, under the influence of the electrostatic force, the upper electrode plate will be attracted and move down to attach to the contacts, making the relay conduct. The substrate of the relay is made of high-resistivity silicon.

The basic procedure for fabrication of the upper electrode plate is sputtering of a gold seed layer onto a polyimide sacrificial layer, followed by plating. The thermal expansion coefficient of polyimide $\left(\alpha_{\mathrm{s}}\right)$ is $70 \times 10^{-6} \mathrm{~K}^{-1}$, while that of gold $\left(\alpha_{\mathrm{f}}\right)$ is

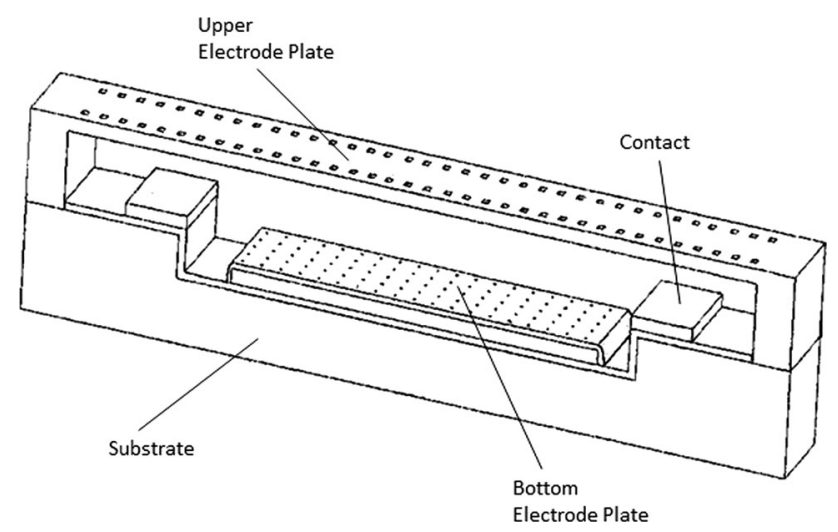

Fig. 1. Schematic structural view of bridge membrane MEMS relay.

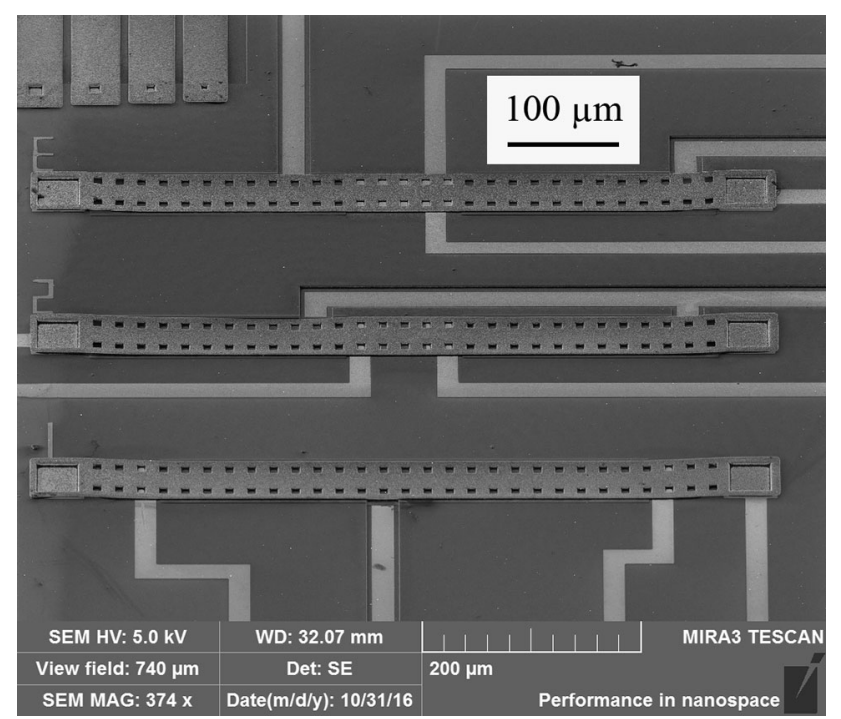

$14.2 \times 10^{-6} \mathrm{~K}^{-1}$, thus $\sigma_{\text {th }}$ is negative according to Eq. 1, meaning that the thermal stress is compressive.

Such compressive stress may lead to film bending and warping, as well as separation or breakage between the membrane and substrate. In terms of the bridge membrane structure, the most crucial effect of residual stress is increase of the driving force and driving voltage. Alberto and Ballestra considered the role of residual stress and its influence on the driving voltage. ${ }^{12}$ They investigated various typical bridge relays and concluded that residual stress of $30 \mathrm{MPa}$ could increase the driving voltage to $56 \mathrm{~V}$ from a value of $27 \mathrm{~V}$, dramatically decreasing the relay sensitivity. Moreover, residual stress will shorten the relay service life.

\section{STRESS MODEL OF BRIDGE MEMBRANE MEMS RELAY}

Nanoindenters are commonly used to study the mechanical properties of MEMS structures, such as 
micro-nano devices and thin-film coatings. ${ }^{13}$ To determine the hardness, plastic modulus, and residual stress of a sample, a standard nanoscale indenter is pressed into the sample surface to determine the relationship between the height $h$ and load $P .{ }^{14}$

\section{Fixed-Fixed Beam Model Without Residual Stress}

Three degrees of freedom (DOF) are limited by the anchor points at both ends of the bridge membrane MEMS relay, thus the membrane of the relay can be simplified to a double-end-fixed beam structure. When loaded, the beam starts to bend and deform elastically under the load. The load-deflection curve of the beam can then be obtained, with the bending deflection on the $x$-axis and the applied load on the $y$-axis.

As shown in Fig. 3, the membrane model used for the upper plate was a double-end-fixed beam with load of $P$ applied at point B. By segmental integration of the differential equation for the deflection in parts $A B$ and $B C$, the deflection at point $B$ is obtained as

$$
\omega=\frac{P x^{3}(l-x)^{3}}{3 E I x^{3}},
$$

where $l$ is the length of the beam, $x$ is the distance between the fulcrum A and loading point B, and $I$ and $E$ represent the cross-sectional moment and plastic modulus of the beam, respectively. Because of the symmetry of the relay and to simplify the model, the force $P$ is applied at the center of the beam, i.e., at $x=l / 2$. It is then clear that the center point $B$ is the point of maximum load, having the maximum deflection of

$$
\omega=\frac{P l^{3}}{192 E I} .
$$

Equation 5 illustrates that the load-deflection curve of the fixed-fixed beam (with center load and no residual stress) is a straight line through the origin. The slope of the curve is equal to the relay's elastic modulus:

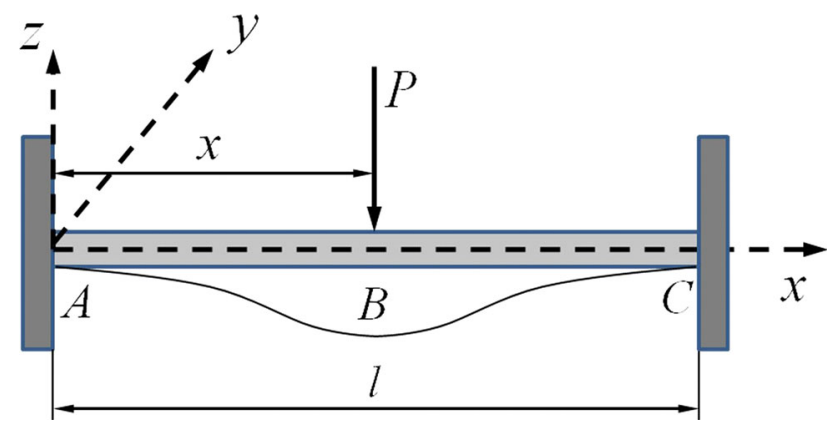

Fig. 3. Double-end-fixed beam with center load.

$$
k=\frac{192 E I}{l^{3}} .
$$

Because of fabrication limitations, bridge membrane MEMS relays are usually designed to have a rectangular beam with cross-sectional moment

$$
I=\frac{b h^{3}}{12},
$$

where $b$ is the beam width and $h$ is the beam thickness. Taking the damping holes in the relay into account, $b$ is given by a linear superposition for the equivalent width. From Eqs. 6 and 7, the elastic modulus of a bridge membrane MEMS relay without consideration of residual stress is

$$
k=\frac{16 E S h^{2}}{l^{3}},
$$

where $S=b h$ is the cross-sectional area.

\section{Tensile Line Model with Residual Stress}

When the equivalent residual stress is applied along the length of a double-end-fixed beam model, the bridge membrane MEMS relay can be simplified using a tensile line model. When the relay is released, the DOF in the thickness direction ( $z$-axis) is freed and the residual stress in this direction disappears, so residual stresses only exist in the length ( $x$-axis) and width (y-axis) directions. This means that the beam is in a double-stress condition. According to Hooke's law, ${ }^{10}$

$$
\left\{\begin{array}{l}
\varepsilon_{x}=\left(\sigma_{x}-v \sigma_{y}\right) / E \\
\varepsilon_{y}=\left(\sigma_{y}-v \sigma_{x}\right) / E \\
\sigma_{z}=0,
\end{array}\right.
$$

where $\sigma_{x}, \sigma_{y}$, and $\sigma_{z}$ represent the stresses in the length, width, and thickness direction, respectively, $\varepsilon_{x}$ and $\varepsilon_{y}$ are the strains in the length and width direction, while $E$ is the plastic modulus of the beam. In isotropic conditions,

$$
\left\{\begin{array}{l}
\varepsilon_{x}=\varepsilon_{y}=\varepsilon \\
\sigma_{x}=\sigma_{y}=\sigma_{\mathrm{re}}
\end{array}\right.
$$

where $\sigma_{\mathrm{re}}$ is the value of the residual stress. Combining Eqs. 9 and 10, we arrive at

$$
\sigma_{\text {re }}=\frac{E}{1-v},
$$

which means that the relationship between the double-axis stress $\sigma_{\text {re }}$ and the equivalent single-axis stress in the $x$-axis direction can be described as

$$
\sigma_{\mathrm{eq}}=\sigma_{\mathrm{re}}(1-v) .
$$

Figure 4 shows a beam with residual stress $\sigma_{\text {re }}$ deformed by a center load $P .{ }^{15}$ Compared with the residual stress-free situation, the beam length is increased by 


$$
\Delta l=l\left(\frac{1}{\cos \theta}-1\right) \approx \frac{1}{2} l \theta^{2}=2 \frac{\omega^{2}}{l},
$$

where $\theta$ is the deflection angle at both beam ends. In the presence of residual stress, the system not only needs to provide the elastic deflection energy for the whole beam but also the extra work required to overcome the stress. The excess energy consumed is

$$
\Delta E=-\sigma_{\mathrm{eq}} S \Delta l
$$

For given deflection, the load $P$ must therefore be increased by $\Delta P$ to offset the work done to overcome the residual stress. When $\Delta P$ is applied, the work done by the variable force is

$$
\Delta W=\frac{1}{2} \Delta P \omega .
$$

Because of conservation of energy, one has

$$
\Delta E=\Delta W .
$$

Combining Eqs. 12-16, one arrives at

$$
\Delta P=-\frac{4 \sigma_{\mathrm{re}}(1-v) S}{l} \omega,
$$

which shows that, under the influence of residual stress, the elastic coefficient of the relay $k^{\prime}$ is increased by

$$
\Delta k=k^{\prime}-k=-\frac{4 \sigma_{0}(1-\sigma) S}{l} .
$$

\section{Formula for Residual Stress}

A finite-element method (FEM) model was established in ANSYS. The elastic coefficient neglecting the effect of residual stress $(k)$ was obtained by

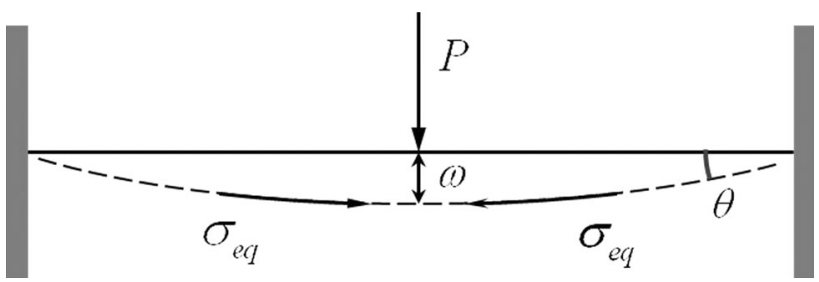

Fig. 4. Schematic diagram of beam deformation static analysis, while its value under residual stress $\left(k^{\prime}\right)$ was obtained using a nanoindenter. The residual stress of the relay can thus be obtained as

$$
\sigma_{\mathrm{re}}=\frac{\left(k-k^{\prime}\right) l}{4 S(1-v)} .
$$

Damping holes are usually spread across the relay, making it difficult to measure the crosssectional area. Therefore, one must combine Eqs. 8 and 9 to eliminate the parameter $S$. Replacing $S$ in Eq. 19 yields

$$
\sigma_{\mathrm{re}}=\frac{4\left(k-k^{\prime}\right) h^{2}}{k l^{2}(1-v)}
$$

\section{MEASUREMENTS WITH THE NANOINDENTER}

\section{Samples and Device}

The sample used in this work was the bridge membrane MEMS relay shown in Fig. 2. Its residual stress was also measured. Its characteristic parameters (Table I) were obtained by using an electron microscope.

The XP nanoindenter used in the experiment (Fig. 5) was produced by the company MTS. This highly automated device was controlled using a computer. In the vertical direction, a strut is moved by the ampere force generated in a coil (while the coil is electrified and placed in a magnetic field). In this way, the load applied by the strut can be adjusted by changing the coil current. At the same time, a differential capacitive displacement sensor measures the displacement of the strut. ${ }^{16}$ The standard Berkovich tip used in the test has triangular-pyramid shape. The angle between the pyramid face and its center line is $65.3^{\circ}$, the angle between the pyramid edge and the center line is $77.05^{\circ}$, while the bottom side length-to-depth ratio is 7.5313. The MTS-XP nanoindenter has maximum indentation depth above $500 \mu \mathrm{m}$, displacement res-

\begin{tabular}{|c|c|c|c|c|}
\hline Parameter & Length $(l)$ & Width $(b)$ & Thickness $(h)$ & Distance between top and bottom plate \\
\hline Designed value $(\mu \mathrm{m})$ & 680 & 50 & 4.8 & 4 \\
\hline Measured value $(\mu \mathrm{m})$ & 689.5 & 49.51 & 4.786 & 3.752 \\
\hline Elastic modulus $(E)$ & $79.5 \mathrm{GPa}$ & & & \\
\hline Poisson's ratio $(v)$ & 0.42 & & & \\
\hline
\end{tabular}
olution below $0.01 \mathrm{~nm}$, maximum load of $500 \mathrm{mN}$, and load resolution of $50 \mathrm{nN}$, making it highly suited for testing of mechanical performance parameters (such as nanohardness, elastic modulus, and yield strength) of solid and membranous materials on nanometer scale.

Table I. Characteristic parameters of relay upper electrode plate 


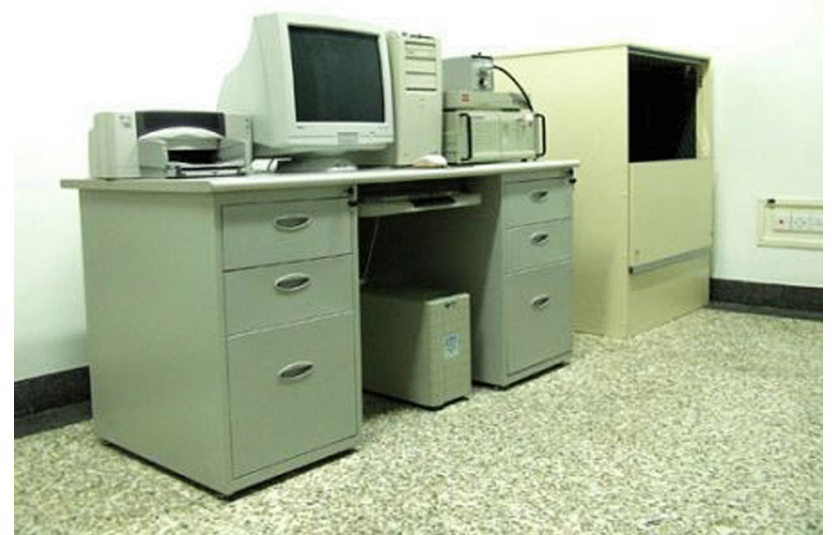

Fig. 5. MTS-XP nanoindenter.

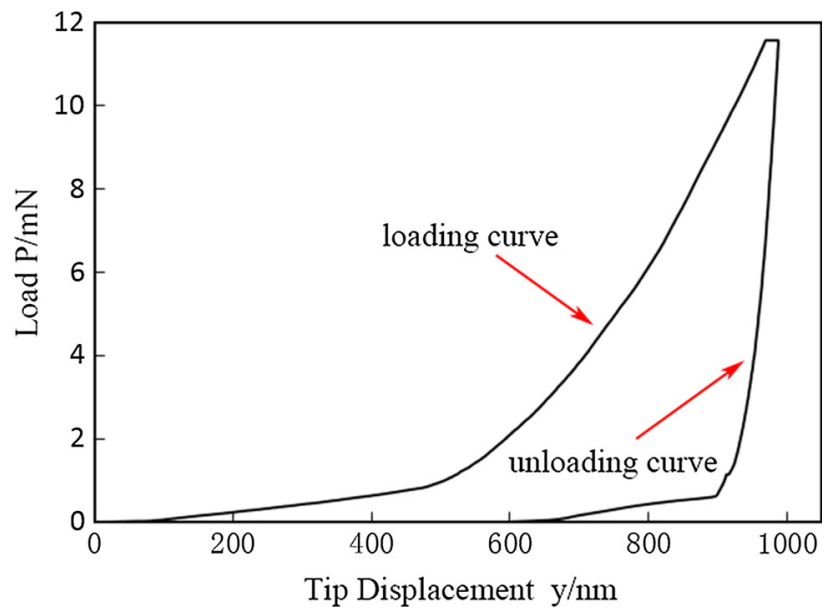

Fig. 6. Load-displacement curve of relay.

\section{Testing Method}

Continuous stiffness measurement (CMS), an optional component of the MTS-XP nanoindenter, is a new dynamic contact method for measuring stiffness and can yield curves for the continuous variation of the stiffness and elastic modulus with indentation depth. ${ }^{16}$ In this test method, the upper electrode plate of the relay is a cantilever, rather than fixed on a rigid substrate, so when loaded it will bend down. In other words, the output indentation depth is actually the tip displacement in the vertical direction. In this way, CMS can provide a curve for the continuous change of the tip displacement with load, and output the continuous changes of stiffness and modulus based on a rigid substrate model. This method can record the whole compression process to obtain relatively comprehensive information.

Before the test, the MTS-XP nanoindenter should be calibrated using a molten silicon reference sample (in accordance with standard ISO-14577) until it

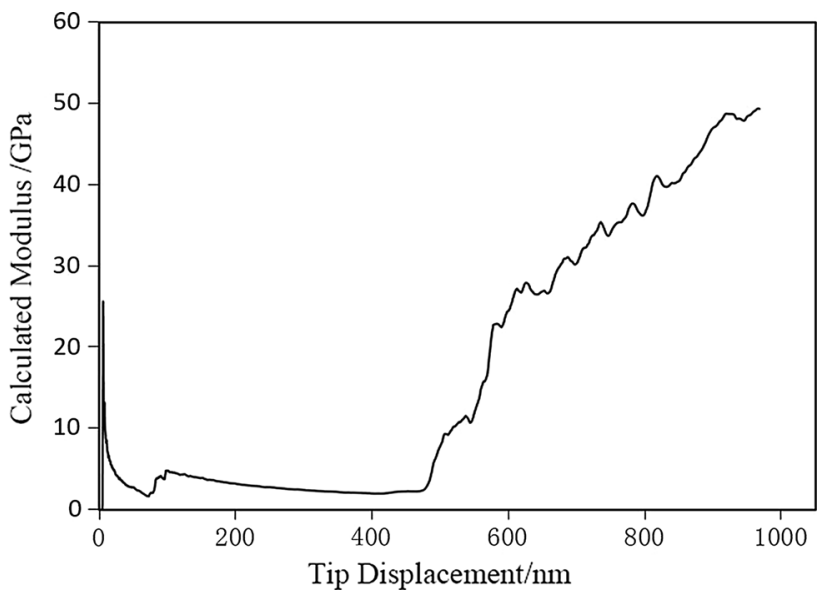

meets the requirement. The sample MEMS relay chip was pasted onto a metal block using resin, then fixed tightly onto the locating platform after horizontally adjusting the sample surface. The mechanical center position of the relay was found by a pressure test to reduce systematic errors due to the structural asymmetry of the relay or needle tip draft. The first step in this process is to apply a load of $5 \mathrm{mN}$ on the relay and move it from one end to the other along the relay axis. The center position shows the largest deflection in the length direction. In the same way, a $5 \mathrm{mN}$ force is then moved in the width direction along a line through the center position of the length direction. This allows the center position of the relay to be found. ${ }^{15}$

Using the CMS method, the tip of the nanoindenter is first aimed at the center position of the relay, then a load of $5 \mathrm{mN}$ is exerted and moved along its center axis in the length direction. It begins to unload when the tip displacement reaches $1 \mu \mathrm{m}$. The CMS method is used to continuously record the curve of the load versus tip displacement.

\section{RESULTS AND ANALYSIS WITH CMS}

\section{Calculation of Residual Stress}

Many samples from the same batch were tested using the MTS-XP nanoindenter in CMS mode to obtain the experimental curve of load versus displacement at the relay center point.

In addition, the CMS method also provides results on the continuous stiffness and modulus based on a rigid substrate model. Although this model is no longer directly applicable, as the upper electrode plate is a cantilever, the data can still be used as reference values. The calculated modulus-displacement curve is shown in Fig. 7.

When a small force is applied, the upper electrode of the relay is suspended rather than pressed into it. The tip will bend the whole beam under elastic deflection. As seen in Fig. 7, the calculated modulus in the corresponding stage is flat with a small value. 
At this time, the tip displacement $y$ equals the deflection of the beam $\omega$. If the curve for the flat stage in Fig. 6 is magnified (as shown in Fig. 8), it is clear that the load-displacement curve is approximately linear, with displacement changing from $100 \mathrm{~nm}$ to $400 \mathrm{~nm}$.

Since the indenter tip is not ideal (even a new one still has radius of curvature of approximately $20 \mathrm{~nm}$ ), the data between depths of $0 \mathrm{~nm}$ and $20 \mathrm{~nm}$ will be invalid. In this paper, data with larger error obtained at depth lower than $100 \mathrm{~nm}$ were rejected. Fitting a line to Fig. 8 from $100 \mathrm{~nm}$ to $400 \mathrm{~nm}$ (using the least-squares method) yields the slope $k^{\prime}$, i.e., the elastic modulus when considering the residual stress, as

$$
k^{\prime}=1.868 \times 10^{-3} \mathrm{mN} / \mathrm{nm} .
$$

When the load is larger, the tip of the indenter goes into the upper electrode plate and the relay deflection is mainly due to plastic deflection of the metal under the relay. As can be seen in Fig. 7, the curve then starts to rise sharply, indicating that the tip has pierced the metal. At the same time, the load-displacement curve is no longer a straight line but a quadratic parabola. ${ }^{16}$ When the system starts to unload, the curve is almost vertical to the $x$-axis since plastic displacement is unrecoverable (Fig. 6). Later, the elastic deflection starts to recover and the curve is basically parallel to the load curve.

The relay's elastic modulus, without residual stress, was simulated using the finite-element method (FEM). Figure 9 shows the model and load of the bridge membrane MEMS relay built in FEM software ANSYS. Figure 10 shows the load-deviation curve taking no account of residual stress.

The slope of the curve in Fig. 10 represents the elastic modulus $k$ with no regard for residual stress:

$$
k=1.633 \times 10^{-3} \mathrm{mN} / \mathrm{nm} .
$$

Substituting these values for $k$ and $k^{\prime}$ into Eq. 20, the residual stress of the relay $\sigma_{\text {re }}$ can be calculated to be

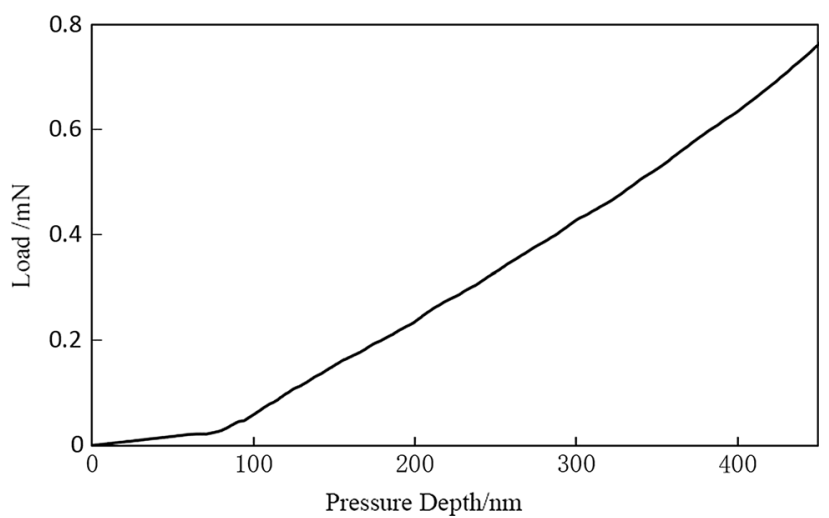

Fig. 8. Load-displacement curve with small load.

$$
\sigma_{\mathrm{re}}=-3.28 \mathrm{MPa} \text {. }
$$

The stress is negative, indicating that it is compressive, a conclusion in accordance with that drawn from the stress generation mechanism. During the production process, the residual stress of gold ranges from $-50 \mathrm{MPa}$ to $60 \mathrm{MPa},{ }^{17}$ hence this experiment result is reasonable. Because the residual stress is compressive and the relay beam is slender, the problem of stability must be considered. When the stress is larger than the buckling critical value, the structure will bifurcate and buckle. The critical stress $\sigma_{\mathrm{rc}}$ is ${ }^{18}$

$$
\sigma_{\mathrm{rc}}=-\frac{\pi^{2} E h^{2}}{3 l^{2}(1-v)} .
$$

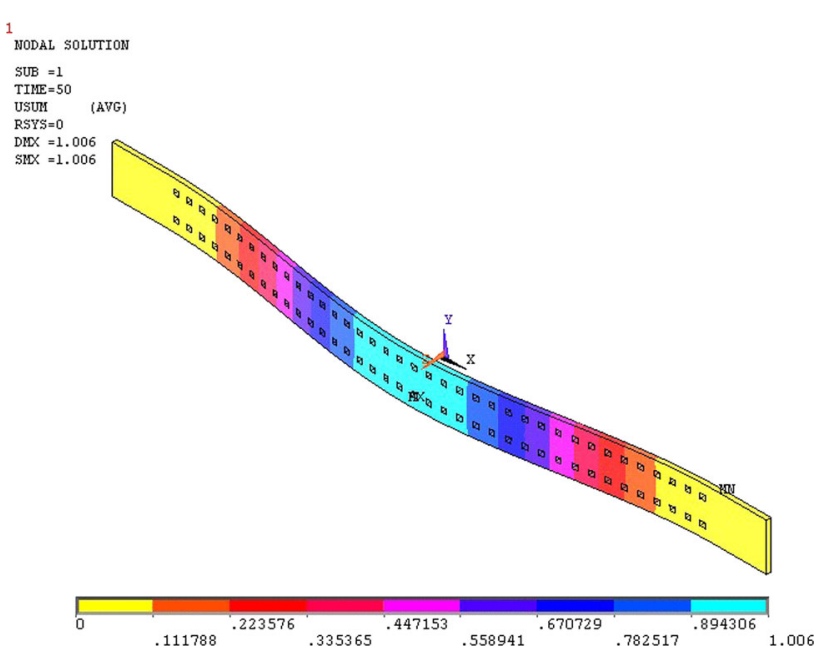

Fig. 9. Three-dimensional model of MEMS relay.

1
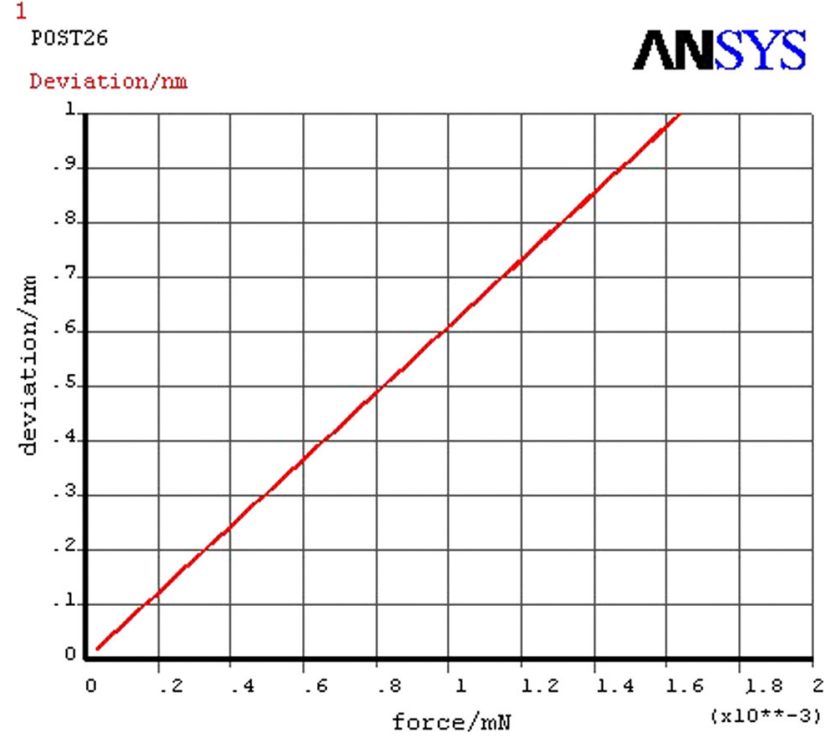

Fig. 10. Load-deviation curve without considering residual stress. 
Table II. Elastic coefficient for an eccentric load

\begin{tabular}{|c|c|c|c|c|c|}
\hline Loading position relative to center point, $(x, y)(\mu \mathrm{m})$ & $\mathbf{0 , 0}$ & 20, 10 & 10,10 & $10,-10$ & $\mathbf{2 0},-\mathbf{1 0}$ \\
\hline Elastic modulus, $k\left(\mathrm{~N} \mathrm{~m}^{-1}\right)$ & 1.663 & 1.599 & 1.631 & 1.630 & 1.600 \\
\hline Relative deviation, $e(\%)$ & 0 & 3.848 & 1.924 & 1.984 & 3.788 \\
\hline
\end{tabular}

We calculate

$$
\sigma_{\mathrm{rc}}=-22.5 \mathrm{MPa}
$$

As shown in Fig. 1, there was no obvious warp in the relay, and the calculated residual stress $\sigma_{\text {re }}$ is also smaller than the critical value.

\section{Error Analysis}

When using a nanoindenter, the tip is located optically. More specifically, the loading position is decided by the device operator using a microscope. However, the tip draft may lead to the tip end being loaded in the wrong position, causing positioning errors. ${ }^{15}$ With the help of the FEM analysis software, the elastic coefficient for an eccentric load was simulated, as presented in Table II.

The results in Table II demonstrate that, when the load is eccentric, the elastic coefficient will only change a little; For example, when the force is applied at the point $(20,10) \mu \mathrm{m}$, the relative deviation is only $3.8 \%$. In addition, the Berkovich tip of the nanoindenter applies the force at one point, which may cause the beam to deflect, meaning that the theoretical model may deviate from the real beam. According to Eq. 20, the length of the relay is significantly larger than its thickness. This means that the accuracy of the thickness measurement will significantly affect the residual stress calculation result. Therefore, the thickness data must be precisely measured by electron microscope to ensure it is as accurate as possible.

\section{CONCLUSIONS}

Based on several simplified mechanical models of a bridge membrane MEMS relay, we propose a method to measure the residual stress (using a nanoindenter, continuous stiffness measurements, and finite-element analysis). The method is easy to carry out, and the data provided are reliable. As a result, it can be used as a reference for design of MEMS devices using surface technology, as well as for measurement and analysis of residual stress. However, it is worth noting that, when the residual stress is larger than the critical value, the modeling and testing method based on bending theory will no longer be applicable, as the relay will undergo structural buckling distortion, requiring further investigation on a stress model and measurement technique using buckling instability theory.

\section{ACKNOWLEDGEMENTS}

This work was performed in The State Key Laboratory of Precision Measurement Technology and Instruments at Tsinghua University, Beijing, China. The authors thank Dr. Shuang-Li YE at Wuhan University for her helpful suggestions. Funding was provided by $\mathrm{Ph}$.D. Programs Foundation of Ministry of Education of China (Grant No. 041503028); Tsinghua University Scientific Research Project independently (Grant No. 52300300916).

\section{REFERENCES}

1. Z. You, H.J. Li, and G.F. Zhang, Piezoelectr. Acoustoopt. 3, 8 (2006).

2. B. Ma, Z. You, Y. Ruan, S.K. Chang, and G.F. Zhang, Microsyst. Technol. 22, 911 (2016).

3. Y.G. Sun, Noise Vib. Control 6, 32 (2007).

4. X. Yan, W. Brown, Y. Li, J. Papapolymerou, C. Palego, J. Hwang, and R. Vinci, J. Microelectromech. Syst. 18, 570 (2009).

5. P.J. Withers and H.K.D.H. Bhadeshia, Mater. Sci. Technol. 17, 355 (2013).

6. S. Chen, T.V. Baughn, Z.J. Yao, and C.L. Goldsmith, J. Microelectromech. Syst. 11, 309 (2002).

7. P. Krulevitch, Diss. Abstr. Int. 55, 253 (1995).

8. H. Windischmann, J. Appl. Phys. 62, 1800 (1987).

9. C. Davis, Thin Solid Films 226, 30 (1993).

10. G.H. Mohamed, MEMS: Design and Fabrication (Boca Raton: CRC/Taylor \& Francis, 2006).

11. Y. Ruan, Z. You, and J.Z. Yang, China Patent No. 200910091536.8 (2010).

12. A. Ballestra, E. Brusa, G. De Pasquale, M.G. Munteanu, and A. Somà, IEEE Trans. Geosci. Remote Sens. 110, 232 (2008).

13. Y. Zhou, M.J. Wang, and C.S. Yang, Mod. Sci. Instrum. 4, 4 (2004).

14. J.N. Ding, Y.G. Meng, and S.Z. Wen, J. Tsinghua Univ. 2, 6 (2001).

15. L.Y. Xu, D.C. Li, and R.P. Liu, Chin. J. Sci. Instrum. 1, 36 (2010).

16. T.H. Zhang, The Introduction and Application of Micro and Nano Mechanical Measurement Technology (Beijing: China Machine Press, 2005).

17. K.J. Cheng and S.Y. Cheng, Prog. Nat. Sci. 6, 5 (1998).

18. J.S. Chen, Z. You, and B. Li, Nanotechnol. Precis. Eng. 9, 16 (2011). 